\title{
Stellar Streams and Clouds in the Galactic Halo
}

\author{
Carl J. Grillmair and Jeffrey L. Carlin
}

\begin{abstract}
Chapter 4 from the volume "Tidal Streams in the Local Group and Beyond: Observations and Implications"; ed. Newberg, H. J., \& Carlin, J. L. 2016, Springer International Publishing, Astrophysics and Space Science Library, 420 ISBN 978-3-319-19335-9; DOI 10.1007/978-3-319-19336-6_1
\end{abstract}

\begin{abstract}
Recent years have seen the discovery of an ever growing number of stellar debris streams and clouds. These structures are typically detected as extended and often curvilinear overdensities of metal-poor stars that stand out from the foreground disk population. The streams typically stretch tens of degrees or more across the sky, even encircling the Galaxy, and range in heliocentric distance from 3 to $100 \mathrm{kpc}$. This chapter summarizes the techniques used for finding such streams and provides tables giving positions, distances, velocities, and metallicities, where available, for all major streams and clouds that have been detected as of January 2015. Sky maps of the streams are also provided. Properties of individual tidal debris structures are discussed.
\end{abstract}

\section{Observational techniques}

With the advent of wide-area, digital sky surveys (primarily the Sloan Digital Sky Survey, SDSS, as well as the 2MASS and WISE surveys), we have seen a remarkable period of discovery in the search for Galactic substructures. From the initial detection of short tidal tails emanating from the globular cluster Palomar 5 in the SDSS commissioning data (Odenkirchen et al. 2001), to the mapping of M-giants in the Sagittarius stream completely around the sky using 2MASS (Majewski et al. 2003), our knowledge of coherent structures in the halo of our Galaxy has advanced spectacularly. While there have been many previous attempts to map large regions of sky in search of tidal streams and substructures, it appears in hindsight that these efforts were largely defeated by insufficient or non-contiguous sky coverage, variable depth or completeness, or insufficiently uniform calibration.

Spitzer Science Center, Mail Stop 220-6, 1200 E. California Blvd., Pasadena, CA 91125, U.S.A.; Rensselaer Polytechnic Institute, 110 8th Street, Troy, NY 12180, U.S.A. 
The streams and clouds described here were all initially detected using photometric techniques. These techniques rely on the separation in color-magnitude space of relatively low-metallicity halo stars from the far larger population of nearby foreground stars. Early efforts to detect tidal debris streams applied these techniques to photographic surveys of regions surrounding globular clusters and dwarf galaxies (Grillmair et al., 1995; Irwin \& Hatzidimitriou, 1995; Leon et al., 2000). The same techniques are now being applied to deeper, wider, and better calibrated large-scale digital surveys (e.g., SDSS, 2MASS, WISE, PAndAS, and Pan-STARRS). Other halo substructures have been detected in radial velocity surveys as cold (low velocity dispersion), coherent subsets of local halo stars; these are described in Chapter 5 .

Early efforts at separating halo populations from foreground stars relied on fairly simple color and magnitude cuts designed to select blue main sequence turn-off stars. This technique was used with some success to find tidal streams emanating from globular clusters (Grillmair et al. 1995, Leon et al. 2000, Odenkirchen et al. 2001). Rockosi et al. (2002) subsequently demonstrated the utility of a matched filter in making better use of the available information and pushing detection limits to considerably lower surface densities. By assigning weights or likelihoods to individual stars based on their photometric uncertainties and the color-magnitude distribution of nearby field stars, the matched filter yields the optimal contrast between different stellar populations. Grillmair and Dionatos (2006a, 2006b) adopted this technique to extend the Pal 5 tidal stream and to discover the tenuous and very cold stream GD-1. More sophisticated techniques are combining photometric selection with radial velocity surveys (e.g., Newberg et al. 2009) to push detection limits to still lower surface densities.

Several stream candidates have been detected purely photometrically as long overdensities in the SDSS and WISE surveys and await confirmation via velocity surveys. Given the low surface densities of known streams, and the fact that stars can only be assigned probabilities of being associated with either the field or the stream, it can be a fairly expensive proposition to obtain velocity information. Telescope time allocation committees are loath to grant large blocks of time when the anticipated yield might be as little as $10 \%$ of the targets surveyed. Confirmation of some of the most tenuous streams may consequently have to wait for results of massively multiplexed spectroscopic surveys such as LAMOST (Cui et al., 2012; Deng et al., 2012; Luo et al., 2012; Zhao et al., 2012) and DESI (Dark Energy Spectroscopic Instrument; formerly known as BigBOSS).

Even while we wait for spectroscopic confirmation, more stream candidates are actively being sought. While tightly constraining the shape and extent of the Galactic potential with tidal streams will ultimately require full, six-dimensional phase space information (e.g. Willett et al. 2009, Koposov et al. 2010, Law \& Majewski 2010), simply finding more streams will enable us to better understand the accretion history of the Galaxy. The distances and orientations of the streams give us some idea of their orbits and the likely distribution of their progenitors. And if we can properly quantify the current detection biases, we will also be in a better position to infer the total population of such streams and their progenitors. 


\section{Currently known stellar debris streams and clouds in the Galactic halo}

Here we present tables that give some basic information for all of the currently known streams and clouds. We define streams as overdensities of stars that are significantly longer than they are wide. Where radial velocities have been measured, they have a narrow line-of-sight velocity dispersion that can vary with position along the stream. Narrower, lower velocity dispersion streams are thought to result from the tidal disruption of globular clusters, while wider, hotter streams are thought to result from the disruption of dwarf galaxies. Clouds, on the other hand, are spatially very large overdensities of halo stars whose origins are currently not understood. These clouds may be the tidal debris from dwarf galaxies that has piled up near the apogalactica of highly eccentric orbits. There is also evidence that at least one cloud is the disrupted remains of the core of the dwarf galaxy.

Tables 1 and 2 are primarily intended to 1) aid non-specialists in identifying unexpected featues in their data, and 2) provide interested researchers with some basic parameters and references to more detailed works. The tables are neither intended nor usable for comparative studies.

Table 1 summarizes the basic properties of all currently known halo debris streams in order of discovery. We do not include globular or open clusters with power-law profiles extending beyond the nominal King tidal radii. Grillmair et al. (1995) and Leon et al. (2000) found such extensions around most of the globular clusters in their respective surveys. Given both these results and theoretical expectations, it is probably fair to say that it would be surprising to find a globular cluster that, if examined sufficiently deeply, did not show evidence of tidal stripping. We therefore list only streams and structures with a projected extent of at least a few degrees on the sky. We report all tidal streams discovered in the literature, including some that remain controversial. For example, the Monoceros Ring could actually be a feature of the Milky Way's stellar disk (see Chapter 3). The Virgo Stellar Stream (VSS) and the Virgo Overdensity (VOD) could be the same structure, or several structures that overlap in configuration space. As several deep, wide-area surveys are in progress in both hemispheres, and as the search for stellar substructures remains quite active, we expect this table to become incomplete fairly quickly.

Column descriptions for Tables 1 and 2 are as follows:

Designation: This column gives the common name by which the particular feature is known in the literature. There exists as yet no convention on how streams should be named and a delightful anarchy has ensued. In cases where the progenitor is known, the feature is quite naturally named after the progenitor (e.g., Sagittarius stream, Pal 5, NGC 5466). Some researchers have named streams after the constellations or regions of the sky in which they were first found, or in which they currently appear strongest (e.g., Monoceros, Cetus Polar Stream, Anticenter Stream, Triangulum/Pisces, Ophiuchus). Still others are named for the surveys in which they were first detected (e.g., ATLAS stream, PAndAS MW stream), after 
some discovery characteristic (Orphan, EBS), or after rivers in Greek mythology (Acheron, Lethe, Cocytos, Styx, Alpheus, Hermus, \& Hyllus).

Progenitor: This column provides the name of the progenitor, if known or suspected. Where there is a question mark, readers are referred to the references for the source of the uncertainty. If the progenitor is listed as dG? (dwarf galaxy) or GC? (globular cluster), the actual progenitor is unknown, but its likely nature is conjectured based on the strength or morphology of the stream. Extensive, broad (FWHM $>500 \mathrm{pc}$ ), or hot streams $\left(\sigma_{v} \geq 10 \mathrm{~km} \mathrm{~s}^{-1}\right)$ presumably arose from more massive systems such as dwarf galaxies, whereas narrower (FWHM $<200 \mathrm{pc}$ ), less populous, and colder streams $\left(\sigma_{v} \leq 5 \mathrm{~km} \mathrm{~s}^{-1}\right)$ most likely arose from globular clusters. This latter conclusion is based primarily on the similarity in the cross-sectional widths of such streams ( $\sim 100 \mathrm{pc}$ ) to those of known globular cluster streams (Pal 5, NGC 5466). On the other hand, Carlberg (2009) has shown that streams should be heated by encounters with other Galactic constituents, and that initially narrow streams should become hotter and broader with time. Our division between globular cluster and dwarf galaxy streams must therefore be regarded with some skepticism until more observational evidence can be brought to bear (e.g. progenitor identifications, $\alpha$-element ratios, etc).

Known Extent: To help researchers to identify structures they may come across in the course of their work, we provide the maximum extent of the streams on the celestial sphere, as determined either in the discovery paper or in subsequent investigations. While the positions and trajectories of some features are shown in Figures 1 through 6 , readers are generally referred to the discovery or follow-up papers for more detailed maps of the streams. In some cases the streams evidently extend beyond the discovery survey regions. For these features we simply provide the R.A. and Dec limits of the survey used.

Distance: This column provides the range of heliocentric distances for different portions of streams. Where different distances are estimated by different authors, we provide only the most recent estimates. These distance estimates will undoubtedly be refined as deeper surveys are carried out, spectroscopic metallicities are obtained, proper motions and Galactic parallaxes are measured, or RR Lyrae can be physically associated with streams. As distances can vary greatly from one portion of a given stream to another, readers are referred to discovery and follow-up papers for estimates of distance with position.

$V_{h e l}$ : If radial velocities have been measured for one or more portions of a stream, the range of velocities is given here. This range can be quite large (e.g., Sagittarius or GD-1), where the length of the stream, combined with the Sun's motion, can produce very substantial gradients over the length of the stream. Once again, readers are referred to the references for more specific information.

$[\mathrm{Fe} / \mathrm{H}]$ : Metallicity estimates can be based on the color-magnitude locus of stream stars, on the metallicity of the matched filter that yields the highest signalto-noise ratio, or on spectroscopy of individual stars. Estimates based solely on photometry can have fairly large uncertainties and are consequently flagged with question marks. 
Selected References: We provide a selected set of references for each stream. These include the discovery paper(s) and subsequent works that demonstrably extend the stream, provide additional spectroscopic or proper motion measurements, examine stream morphology, RR Lyrae content, model the orbits of the streams, or use the streams as probes of the Galactic potential.

Clouds are listed in Table 2, and are distinguished from streams by their more diffuse, non-localized nature. These features are also likely to be the result of tidal stripping or disruption, but determining their nature and origin may have to await future radial velocity and proper motion surveys.

The locations and extent of many of the structures in Tables 1 and 2 are shown in Figures 1 and 2. The PAndAS MW, ATLAS, and Ophiuchus streams are shown separately in Figures 3, 4, and 5. 
Table 1 Currently Known Halo Streams

\begin{tabular}{|c|c|c|c|c|c|c|}
\hline Designation & Progenitor & Known Extent & $\begin{array}{l}\text { Distance } \\
\mathrm{kpc}\end{array}$ & $\begin{array}{c}\mathrm{V}_{h e l} \\
\mathrm{~km} \mathrm{~s}^{-1}\end{array}$ & {$[\mathrm{Fe} / \mathrm{H}]$} & Selected References \\
\hline $\begin{array}{l}\text { Sagittarius } \\
\text { Stream }\end{array}$ & $\begin{array}{l}\text { Sagittarius } \\
\text { dSph }\end{array}$ & Circum-sky & $7-100$ & $(-200,+200)$ & $(-1.15,-0.4)$ & $\begin{array}{l}\text { Ibata et al. 1994, Mateo et al. 1998, } \\
\text { Alard 1996, Totten \& Irwin 1998, } \\
\text { Ibata et al. 2001a, b, Majewski et al. 2003, } \\
\text { Martinez-Delgado et al. 2004, } \\
\text { Vivas et al. 2005, Belokurov et al. 2006b, } \\
\text { Fellhauer et al. 2006, Bellazzini et al. 2006b, } \\
\text { Chou et al. 2007, Law et al. 2009, } \\
\text { Law \& Majewski 2010, Keller et al. } 2010 \\
\text { Carlin et al. 2012a, Koposov et al. } 2012\end{array}$ \\
\hline $\begin{array}{l}\text { Virgo Stellar } \\
\text { Stream }\end{array}$ & NGC 2419? & $\begin{array}{c}180^{\circ}<\text { R.A. }<195^{\circ} \\
-4^{\circ}<\delta<0^{\circ}\end{array}$ & 19.6 & 128 & -1.78 & $\begin{array}{l}\text { Vivas et al. 2001, Duffau et al., 2006, } \\
\text { Newberg et al. 2007, Duffau et al. } 2014\end{array}$ \\
\hline Palomar 5 & Palomar 5 & $\begin{aligned} 225^{\circ} & <\text { R.A. }<250^{\circ} \\
-3^{\circ} & <\delta<8.5^{\circ}\end{aligned}$ & 23 & -55 & -1.43 & $\begin{array}{l}\text { Odenkirchen et al. 2001, 2003, 2009, } \\
\text { Rockosi et al. 2002, } \\
\text { Grillmair \& Dionatos 2006a, } \\
\text { Carlberg, Grillmair, \& Hetherington } 2012\end{array}$ \\
\hline Monoceros Ring & $\mathrm{dG} ?$ & $\begin{array}{c}108^{\circ}<\text { R.A. }<125^{\circ} \\
-3^{\circ}<\delta<-41^{\circ}\end{array}$ & $\approx 10.5$ & $\approx 100$ & -0.8 & $\begin{array}{l}\text { Newberg et al. 2002, Yanny et al. 2003, } \\
\text { Ibata et al. 2003, Rocha-Pinto et al. 2003, } \\
\text { Penarrubia et al. 2005, Li et al. } 2012 \\
\text { Slater et al. } 2014\end{array}$ \\
\hline NGC 5466 & NGC 5466 & $\begin{array}{c}182^{\circ}<\text { R.A. }<224^{\circ} \\
21^{\circ}<\delta<42^{\circ}\end{array}$ & 17 & 108 & -2.2 & $\begin{array}{l}\text { Belokurov et al. 2006a, } \\
\text { Grillmair \& Johnson 2006, } \\
\text { Fellhauer et al. 2007b, Lux et al. } 2012\end{array}$ \\
\hline Orphan Stream & $\mathrm{dG} ?$ & $\begin{array}{c}143^{\circ}<\text { R.A. }<165^{\circ} \\
-17^{\circ}<\delta<+48^{\circ}\end{array}$ & $20-55$ & $(95,240)$ & -2.1 & $\begin{array}{l}\text { Grillmair 2006a, Belokurov et al. 2007a } \\
\text { Fellhauer et al. 2007a, Sales et al. 2008, } \\
\text { Newberg et al. 2010, Sesar et al. } 2013 \\
\text { Casey et al. } 2013\end{array}$ \\
\hline GD-1 & GC? & $\begin{array}{c}134^{\circ}<\text { R.A. }<218^{\circ} \\
14^{\circ}<\delta<58^{\circ}\end{array}$ & $7-10$ & $(-200,+100)$ & -2.1 & $\begin{array}{l}\text { Grillmair \& Dionatos 2006b, } \\
\text { Willett et al. 2009, } \\
\text { Koposov, Rix, \& Hogg 2010, } \\
\text { Carlberg \& Grillmair } 2013\end{array}$ \\
\hline AntiCenter Stream & dG? & $\begin{array}{c}121^{\circ}<\text { R.A. }<130^{\circ} \\
-3^{\circ}<+63^{\circ}\end{array}$ & $\approx 8$ & $(50,90)$ & -0.96 & $\begin{array}{l}\text { Grillmair 2006b, } \\
\text { Grillmair, Carlin, \& Majewski 2008, } \\
\text { Li et al. } 2012\end{array}$ \\
\hline EBS & GC? & $\begin{aligned} 132^{\circ} & <\text { R.A. }<137^{\circ} \\
-3^{\circ} & <\delta<16^{\circ}\end{aligned}$ & 10 & $(71,85)$ & -1.8 & $\begin{array}{l}\text { Grillmair 2006b, Grillmair 2011, } \\
\text { Li et al. } 2012\end{array}$ \\
\hline Acheron & GC? & $\begin{array}{c}232^{\circ}<\text { R.A. }<258^{\circ} \\
3^{\circ}<\delta<20^{\circ}\end{array}$ & $3.5-3.8$ & & $-1.7 ?$ & Grillmair 2009 \\
\hline Cocytos & GC? & $\begin{aligned} 197^{\circ} & <\text { R.A. }<257^{\circ} \\
8^{\circ} & <\delta<30^{\circ}\end{aligned}$ & 11 & & $-1.7 ?$ & Grillmair 2009 \\
\hline Lethe & GC? & $\begin{array}{c}176^{\circ}<\text { R.A. }<252^{\circ} \\
22^{\circ}<\delta<37^{\circ}\end{array}$ & 13 & & $-1.7 ?$ & Grillmair 2009 \\
\hline Styx & Bootes III? & $\begin{array}{c}201^{\circ}<\text { R.A. }<250^{\circ}, \\
21^{\circ}<\delta<31^{\circ}\end{array}$ & 45 & & $-2.2 ?$ & Grillmair 2009 \\
\hline $\begin{array}{l}\text { Cetus Polar } \\
\text { Stream }\end{array}$ & NGC 5824? & $\begin{array}{l}19^{\circ}<\text { R.A. }<37^{\circ} \\
-11^{\circ}<\delta<+39^{\circ}\end{array}$ & $24-36$ & $(-200,-160)$ & -2.1 & $\begin{array}{l}\text { Newberg, Yanny, \& Willett 2009, } \\
\text { Yam et al. } 2013\end{array}$ \\
\hline $\begin{array}{l}\text { Pisces/ } \\
\text { Triangulum }\end{array}$ & GC? & $\begin{aligned} & 21^{\circ}<\text { R.A. }<24^{\circ} \\
& 23^{\circ}<\delta<40^{\circ}\end{aligned}$ & 35 & 120 & -2.2 & $\begin{array}{l}\text { Bonaca et al. 2012b, Martin et al. 2013, } \\
\text { Martin et al. } 2014\end{array}$ \\
\hline Alpheus & NGC $288 ?$ & $\begin{array}{l}22^{\circ}<\text { R.A. }<28^{\circ} \\
-69^{\circ}<\delta<45^{\circ}\end{array}$ & $1.6-2.0$ & & $-1.0 ?$ & Grillmair et al. 2013 \\
\hline ATLAS stream & Pyxis? & $\begin{array}{l}18^{\circ}<\text { R.A. }<30^{\circ} \\
-32^{\circ}<\delta<-25^{\circ}\end{array}$ & 20 & & $-1.4 ?$ & Koposov et al. 2014 \\
\hline $\begin{array}{l}\text { PAndAS MW } \\
\text { Stream }\end{array}$ & dG? & $\begin{array}{c}0^{\circ}<\text { R.A. }<22^{\circ} \\
40^{\circ}<\delta<48^{\circ}\end{array}$ & 17 & 127 & $-1.5 ?$ & Martin et al. 2014 \\
\hline Hermus & $\mathrm{GC} ?$ & $\begin{aligned} 241^{\circ} & <\text { R.A. }<254^{\circ} \\
5^{\circ} & <\delta<50^{\circ}\end{aligned}$ & 18.5 & & $-2.3 ?$ & Grillmair 2014 \\
\hline Hyllus & GC? & $\begin{aligned} & 245^{\circ}<\text { R.A. }<249^{\circ} \\
& 11^{\circ}<\delta<34^{\circ}\end{aligned}$ & 20 & & $-2.3 ?$ & Grillmair 2014 \\
\hline Ophiuchus stream & $\mathrm{GC}$ & $\begin{aligned} 241^{\circ}<\text { R.A. }<243^{\circ} \\
-7.2^{\circ}<\delta<6.7^{\circ}\end{aligned}$ & $8-9.5$ & 290 & -1.95 & Bernard et al. 2014, Sesar et al. 2015 \\
\hline
\end{tabular}


Table 2 Currently Known Halo Clouds

\begin{tabular}{|c|c|c|c|c|c|c|}
\hline Designation & Progenitor & Known Extent & $\begin{array}{l}\text { Distance } \\
\text { kpc }\end{array}$ & $\begin{array}{c}\mathrm{V}_{\text {hel }} \\
\mathrm{km} \mathrm{s}^{-1}\end{array}$ & {$[\mathrm{Fe} / \mathrm{H}]$} & Selected References \\
\hline $\begin{array}{l}\text { Triangulum- } \\
\text { Andromeda (TriAnd1) }\end{array}$ & dG? & $\begin{aligned}-10^{\circ} & <\text { R.A. }<30^{\circ} \\
20^{\circ} & <\delta<45^{\circ}\end{aligned}$ & 20 & $(-200,-50)$ & -0.6 & $\begin{array}{l}\text { Majewski et al. 2004, Rocha-Pinto et al. 2004, } \\
\text { Martin et al. 2007, Chou et al. } 2011 \\
\text { Deason et al. 2014, Sheffield et al. } 2014\end{array}$ \\
\hline Hercules-Aquila & $\mathrm{dG}$ ? & $\begin{aligned} 0^{\circ} & <l<80^{\circ} \\
-50^{\circ} & <b<50^{\circ}\end{aligned}$ & $10-25$ & $(-130,-120)$ & $(-2.2,-1.4)$ & $\begin{array}{l}\text { Belokurov et al. 2007b, Watkins et al. } 2009 \text {, } \\
\text { Sesar et al. 2010a, Simion et al. } 2014\end{array}$ \\
\hline $\begin{array}{l}\text { Virgo } \\
\text { Overdensity }\end{array}$ & dG? & see map in Figure 3 & $\sim 6-20$ & $(200,360)$ & $(-2.0,-1.0)$ & $\begin{array}{l}\text { Vivas et al. 2001, Newberg et al. 2002, } \\
\text { Juric et al. 2008, Bonaca et al. 2012a, } \\
\text { Carlin et al. 2012b, Duffau et al. } 2014\end{array}$ \\
\hline $\begin{array}{l}\text { Triangulum } \\
\text {-Andromeda } 2 \text { (TriAnd2) }\end{array}$ & dG? & $\begin{array}{c}3^{\circ}<\text { R.A. }<23^{\circ} \\
28^{\circ}<\delta<42^{\circ}\end{array}$ & 28 & $(-200,-50)$ & -0.6 & Martin et al. 2007, Sheffield et al. 2014 \\
\hline Pisces Overdensity & $\mathrm{dG}$ ? & $\begin{array}{l}350^{\circ}<\text { R.A. }<360^{\circ} \\
-1.3^{\circ}<\delta<+1.3^{\circ}\end{array}$ & $80-100$ & -75 & -1.5 & $\begin{array}{l}\text { Sesar et al. 2007, Watkins et al. 2009, } \\
\text { Kollmeier et al. 2009, Sesar et al. 2010b, } \\
\text { Sharma et al. 2010, Vivas et al. } 2011\end{array}$ \\
\hline
\end{tabular}


In the remainder of this chapter we discuss some of the particulars of individual streams listed in Tables 1 and 2 As the Sagittarius and Monoceros/Anticenter streams are the subject of much study and controversy, they merit more detailed discussion, and we refer the reader to Chapters 2 and 3 for more on these systems.

\subsection{Streams with known or likely globular cluster progenitors}

Pal 5: The stream associated the globular cluster Palomar $5(\mathrm{Pal} 5)$ is one of the most striking examples among the known tidal streams. The stream is clearly visible as a narrow ribbon of stars arcing across the corner of SDSS starcount maps such as Figure 1 and the "Field of Streams" from Belokurov et al. (2006b; also reproduced in Chapter 1 of this volume). As it is clearly associated with the cluster, is a welldefined, kinematically-cold stream, and is at a distance $(\sim 23 \mathrm{kpc})$ close enough to be studied in detail, this stream has been the object of intense scrutiny. Indeed, as it can be studied in such exquisite detail, the Pal 5 stream has become something of a test case for models of tidal disruption (e.g., Dehnen et al. 2004; MastrobuonoBattisti et al. 2012) and evolution of the tidal tails in the Galactic halo. The stream is clearly defined over $>25^{\circ}$ on the sky, with radial velocity members now identified over at least $\sim 20^{\circ}$ of its extent (e.g., Odenkirchen et al. 2009; Kuzma et al. 2015). As it is robustly detected above the background stellar density, the Pal 5 stream has been used to explore gaps in streams and their implications for the density of dark matter subhalos (Carlberg et al. 2012; see also Chapter 7). As much of the stream is still in the vicinity of Pal 5, with a rather periodic distribution of clumps near the cluster, there remains some controversy concerning the extent to which these gaps may be a product of epicyclic motions of stars within the stream (Küpper et al. 2008, 2012) rather than the result of encounters with subhalos.

NGC 5466: The tidal stream associated with the globular cluster NGC 5466 has been suggested (Lux et al., 2012) to have strong implications for the shape of the Galactic dark matter halo. Though this stream is quite strong near the cluster (Belokurov et al., 2006a), its more distant reaches are very much more tenuous (Grillmair \& Johnson, 2006). The analysis by Lux et al. (2012) suggested that the trajectory of the northern tip of the stream may have important consequences for the inferred shape of the dark matter halo. More definitive results await the identification of tracers with which to derive velocity and distance to the stream at large separations from the main body of the cluster.

GD-1: Now traced to over $80^{\circ}$ across the northern SDSS footprint, the "GD-1" stream (Grillmair \& Dionatos, 2006b) is the longest of the cold streams discovered to date. At a mean distance of $\sim 9 \mathrm{kpc}$, the stream's narrow $\sim 0.5^{\circ}$ width corresponds to only $\sim 70 \mathrm{pc}$ in cross section, suggesting that the GD-1 progenitor was (or is) a globular cluster. The orbit of this stream has been well established using SDSS velocity measurements by Willett et al. (2009), who found a best-fitting orbit with pericenter of $\sim 14 \mathrm{kpc}$, apocenter of $\sim 28 \mathrm{kpc}$, and low inclination $\left(i \sim 35^{\circ}\right)$. Koposov et al. (2010) explored the constraints that this system places on the shape 
and strength of the Galactic potential, and were able to put tight limits on the circular velocity at the Sun's radius $\left(\mathrm{V}_{c}=224 \pm 13 \mathrm{~km} \mathrm{~s}^{-1}\right)$. Constraints on the shape of the halo are comparatively weak owing to the relatively low orbital inclination and the strong influence of the Galactic disk. Its relatively high surface density over a large angular swath, kinematically cold population, presumed great age, and lack of a nearby progenitor make GD-1 an excellent candidate to search for the perturbing effects of dark matter subhalos (see Chapter 7). The measurement of $8 \pm 3$ stream gaps over $\sim 8 \mathrm{kpc}$ of GD-1's extent by Carlberg \& Grillmair (2013) suggest that the Milky Way contains some 100 dark matter subhalos with $M>10^{6} \mathrm{M}_{\odot}$ within the apocenter of the GD-1 orbit. Verifying and refining this estimate will require both deeper photometric surveys and better radial velocity sampling of GD-1.

EBS: The Eastern Banded Structure (EBS) is a $\sim 18^{\circ}$-long stellar overdensity discovered in the SDSS by Grillmair (2006a). Because of its proximity and similar distance ( $\sim 10 \mathrm{kpc})$ to the Anticenter Stream (ACS), the EBS was originally thought to be associated with the ACS/Monoceros structures. Grillmair (2011) and Li et al. (2012) showed that EBS is distinct both in metallicity and kinematics from the ACS. Furthermore, Grillmair (2011) associated two velocity structures (ECHOS) of Schlaufman et al. (2009) with EBS and determined that its orbit is likely to be highly eccentric, unlike that of the ACS. A $\sim 2^{\circ}$-wide overdensity of stars within the EBS was also noted by Grillmair (2011), who suggested that this object, dubbed Hydra I, may be the remains of EBS's progenitor. Deeper and wider surveys of the EBS are currently in progress and will hopefully shed more light on the nature and origin of this stream.

Pisces/Triangulum: The Pisces/Triangulum Stellar Stream is a narrow $\left(\sim 0.2^{\circ}\right)$ stream that was found as a $\sim 5.5 \mathrm{kpc}-$ long photometric overdensity by Bonaca et al. (2012b), who dubbed it the "Triangulum Stream" based on its proximity to M33. It was detected independently as a kinematically coherent feature among SDSS spectra by Martin et al. (2013), who called it the "Pisces Stellar Stream" because it resides in Pisces. While Bonaca et al. (2012b) estimated a distance of $26 \pm 4 \mathrm{kpc}$ to the stream based on fitting isochrones of metallicity $[\mathrm{Fe} / \mathrm{H}]=-1.0$ to stars in the structure, Martin et al. (2013) were able to estimate a spectroscopic metallicity of $[\mathrm{Fe} / \mathrm{H}] \approx-2.2$, and revised the distance estimate to the Pisces stream to $35 \pm 3 \mathrm{kpc}$. This stream is prominent in stellar density maps of the PAndAS survey (Martin et al. 2014; reproduced here as Figure 3, see panels 4 and 5), extending the stream several degrees northward of the SDSS footprint. Interestingly, Martin et al. (2014) found that the stream ends abruptly, suggesting either a physical truncation or a sharp distance gradient at the northern end. As no progenitor has yet been identified, and as kinematical data exist only for only one point along the stream, an orbit has not been estimated.

Acheron, Cocytos, Lethe, Hermus, \& Hyllus: These stream candidates are long, narrow, but relatively low signal-to-noise overdensities of low-metallicity stars in the northern footprint of the SDSS survey. No velocity information has yet been gleaned for these streams, but efforts are underway to associate tracers such as blue horizontal branch stars and RR Lyrae with one or more of them. 
Alpheus: This nearby $(<2 \mathrm{kpc})$ stream candidate was discovered by combining the WISE All-Sky and 2MASS catalogs (Grillmair et al., 2013). These authors suggest that, by virtue of distance and orientation, the stream could plausibly have originated in the globular cluster NGC 288 some $20^{\circ}$ away. Deeper surveys and velocity information will be required to test this hypothesis.

Ophiuchus: This stream is somewhat unique among our current sample of streams in that it is apparently highly foreshortened, and is seen nearly end-on from our vantage point. It was detected (Bernard et al., 2014) as an overdensity of metalpoor stars in the Pan-STARRS1 $3 \pi$ survey. Though only $2.5^{\circ}$ long, Sesar et al. (2015) subsequently determined that the Ophiuchus stream is actually $\sim 1.6 \mathrm{kpc}$ in length, appearing foreshortened due to our nearly end-on viewing angle. Their velocity and proper motion measurements indicate that the stream is kinematically cold and on a fairly eccentric orbit with peri- and apocentric distances of 3.5 and $17.5 \mathrm{kpc}$, respectively. To explain the rather short length of the stream given its inferred orbit, Sesar et al. suggested that the progenitor must have suffered a substantial change in its orbit and become disrupted only rather recently ( $\sim 250 \mathrm{Myr}$ ago).

\subsection{Streams with presumed dwarf galaxy progenitors}

Orphan stream: The Orphan stream is a roughly $1-2^{\circ}$-wide stellar stream spanning nearly the entire vertical (declination) extent of the SDSS northern Galactic hemisphere footprint. This stream, so called because its progenitor has not been identified, was discovered at about the same time by Grillmair (2006a) and Belokurov et al. (2007b). Because the stream has fairly low surface brightness, determining the distance and velocity of stream members along its extent is difficult. However, some such measurements have been done, and a few attempts have been made to derive orbits. The first of these, by Fellhauer et al. (2007a), explored the possibility that two objects spatially coincident with the stream - the Ursa Major II (UMaII) dwarf spheroidal and the HI cloud known as Complex A - are physically associated with the stream. They concluded that UMaII is likely the progenitor of the Orphan stream, and predicted a strong velocity gradient along the stream. In contrast, Sales et al. (2008) modeled the disruption of a massive two-component dwarf galaxy on the Orphan stream orbit, and found that UMaII is not likely to be the progenitor, and indeed the progenitor is likely to be almost completely disrupted. A more extensive characterization of the stream from SDSS was conducted by Newberg et al. (2010), who then used the data to derive an orbit for the structure and produce an $\mathrm{N}$-body model of a disrupting satellite on this orbit. Their models suggest that the SDSS debris is part of the leading tail of a disrupting satellite on an eccentric $(e \sim 0.7)$ orbit that carries the dwarf out to Galactocentric distances of $\sim 90 \mathrm{kpc}$.

The Orphan stream has been traced with RR Lyrae stars to a distance of $55 \mathrm{kpc}$ by Sesar et al. (2013), who also identified a strong metallicity gradient along the 


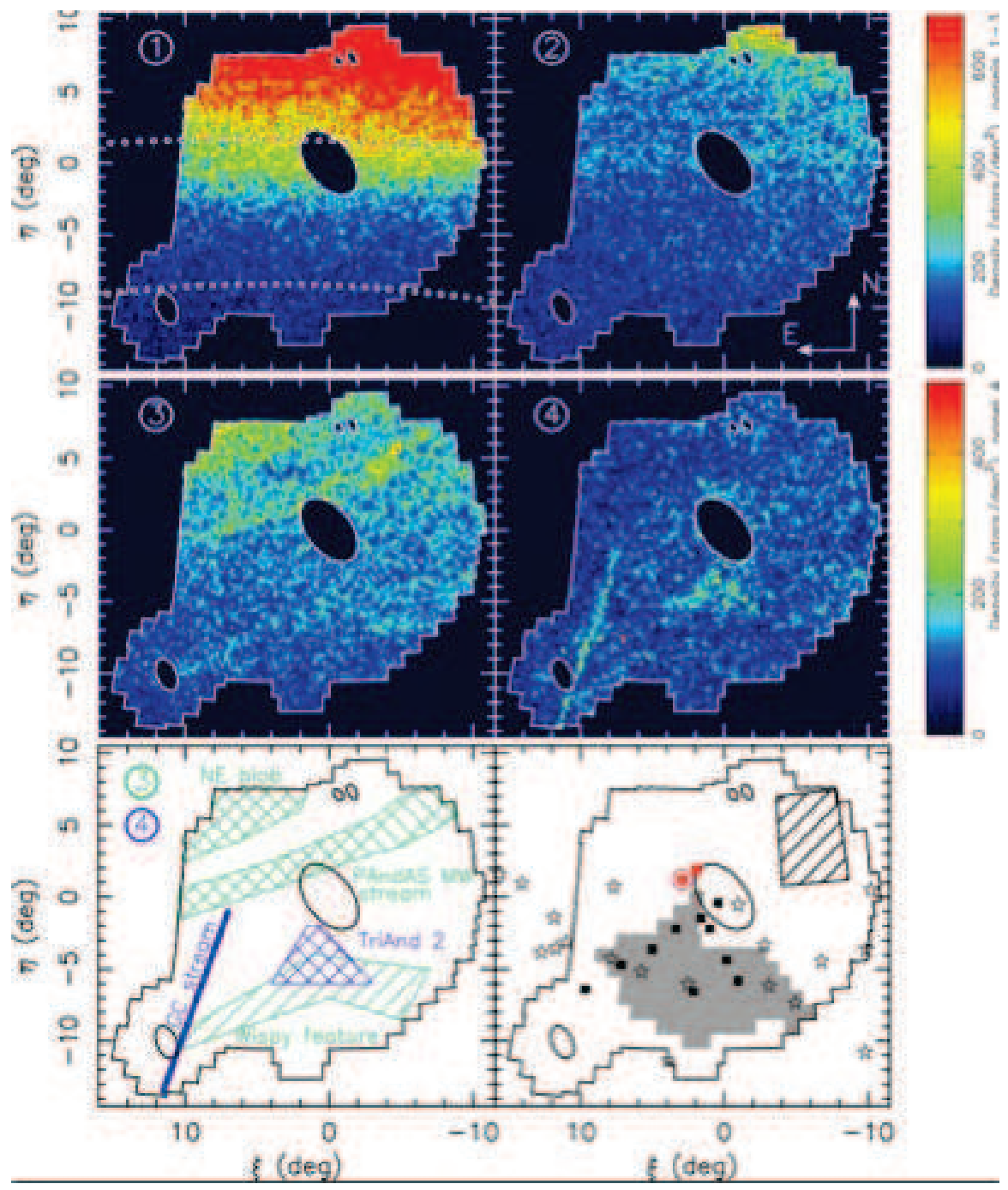

Fig. 3 Stellar density in the PAndAS survey in the foreground of M31 (coordinates are relative to the M31 center, with north upward and east toward the left). Panels 1-4 show smoothed stellar density maps for CMD-filtered stars at mean distances of 7, 11, 17, and $27 \mathrm{kpc}$ from the Sun, respectively. The lower left panel shows the Milky Way substructures identified by Martin et al. (2014) in this so-called "PAndAS Field of Streams." [Figure 2 from Martin et al. (2014).]

stream. These authors could not identify members beyond $55 \mathrm{kpc}$, and suggest that this is either because the leading stream actually ends at that point, or the tracers are lost due to survey incompleteness or low metallicity. The large metallicity spread seen among these RR Lyrae bolsters the case that this is a dwarf galaxy remnant. The low velocity dispersion and large metallicity spread have been confirmed 


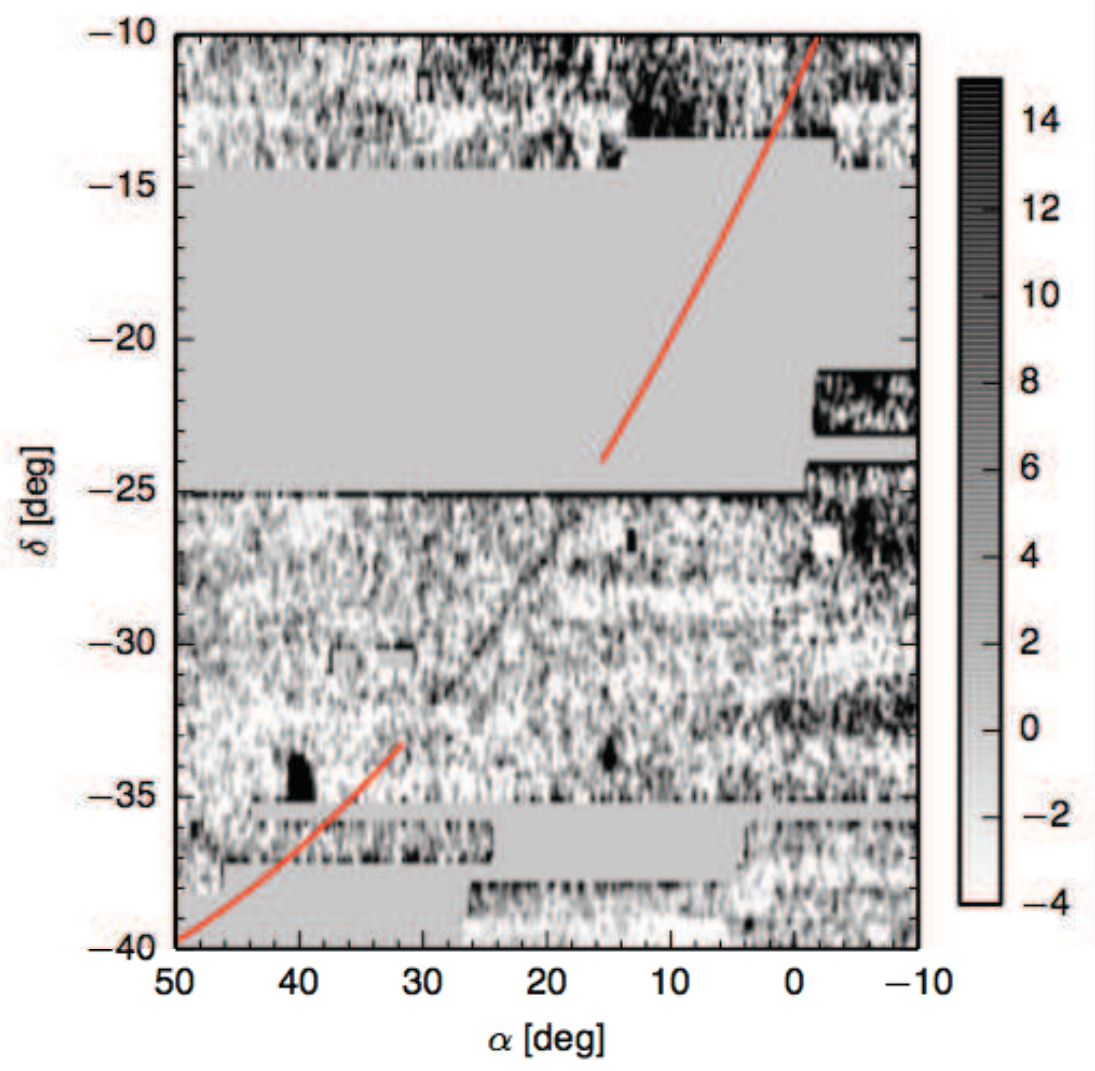

Fig. 4 Surface density map of the ATLAS stream, using a filter optimized for a stellar population with $[\mathrm{Fe} / \mathrm{H}]=-2.1$, an age of $12.5 \mathrm{Gyrs}$, and a distance of $20 \mathrm{kpc}$. Darker shades correspond to higher surface densities. [Figure 1 from Koposov et al. (2014).]

by Casey et al. (2013), and high-resolution spectroscopy (Casey et al., 2014) shows abundances consistent with dwarf spheroidal chemical evolution.

It is unclear whether any of the satellites (UMaII and the Segue 1 dwarf galaxy) or Complex A are associated with the Orphan stream. Further exploration in the southern celestial hemisphere to identify the path of the stream, its stellar density, and possibly a progenitor, is currently underway. We note that this stream is likely to be a particularly good tracer of the Galactic potential, since it is well traced over a large swath (constraining its orbit quite well) and traverses a large range of Galactic radii. The combination of the orbit fit to the Orphan stream by Newberg et al. (2010) and distance constraints from RR Lyrae (Sesar et al. 2013) suggest a fairly low mass for the Galactic halo $\left(\sim 3 \times 10^{11} \mathrm{M}_{\odot}\right.$ within $\left.60 \mathrm{kpc}\right)$.

Cetus Polar Stream: This structure was identified in a study of the Sagittarius (Sgr) stream (see Chapter 2) with SDSS data by Yanny et al. (2009) as a set of BHB 


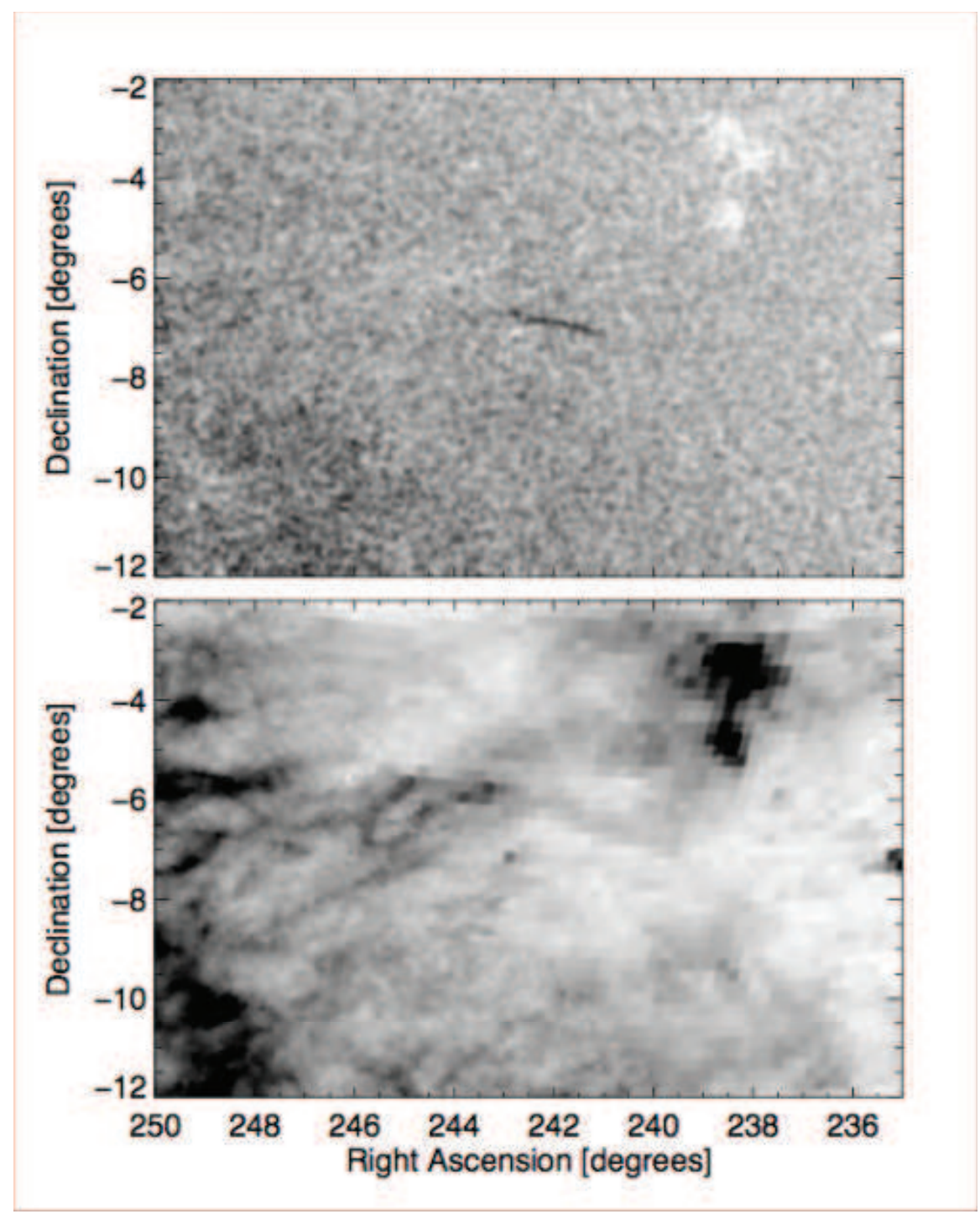

Fig. 5 Surface density map of the Ophiuchus stream, optimized for old and metal-poor stars at a distance of 8-12 kpc. Darker shades correspond to higher surface density. The lower panel shows a reddening map of the same region. [Adapted from Figure 1 of Bernard et al. (2014).]

stars with velocity and metallicity distinct from that of Sgr. Newberg et al. (2009) followed this up by tracing the stream with BHB stars from SDSS DR7, finding a stream aligned roughly along constant Galactic longitude in the south Galactic cap. The authors showed that the ratio of blue straggler stars (BSSs) to BHBs is higher in Sgr than the Cetus Polar Stream (CPS), and that most of the BHB stars in this 
region of the sky (at distances of $\sim 30 \mathrm{kpc}$ ) are associated with the CPS and not Sgr. This was confirmed by Koposov et al. (2012), who used the offset between BHBs and BSSs to separate Sgr and the CPS in the South. The distance and velocity trends seen in this work imply that the CPS progenitor is (or was) on an orbit that counterrotates relative to that of Sgr, as had also been found via orbit-fitting by Newberg et al. (2009). Interestingly, Newberg et al. (2009) determined that the massive globular cluster NGC 5824 lies very close to their computed orbit, at nearly the predicted distance, and with almost exactly the predicted radial velocity. Moreover, the tidal extensions found by Grillmair et al. (1995) and Leon et al. (2000) for NGC 5824 also lie along the predicted orbit of the CPS.

The most extensive examination of the CPS was that of Yam et al. (2013). This group used the additional data available in SDSS DR8 to refine the kinematic signature and the distances to the CPS. The newly-available contiguous sky coverage of DR8 photometry was exploited to map the density of BHB stars along the stream and fit the stream width. Yam et al. then used all of this new information to fit an orbit to the stream, and found a low-eccentricity $(e \approx 0.2)$, highly inclined $\left(i \approx 87^{\circ}\right)$ orbit with apo- and pericentric distances of $\sim 36$ and $\sim 24 \mathrm{kpc}$ from the Galactic center. $N$-body models of $10^{8} M_{\odot}$ satellites (assuming mass follows light) on this orbit were found to reproduce the stream velocities and their dispersions, along with the width of the stream, as a function of position. However, matching the density profile of the stream requires a lower mass satellite of $10^{6} M_{\odot}$. This suggests that mass does not (or did not) follow light in the progenitor of the CPS. In other words, the stream must originate from a low-mass, dark matter-dominated satellite similar to the "ultra-faint dwarf spheroidals" found in SDSS. This is corroborated by the low metallicity $([\mathrm{Fe} / \mathrm{H}] \sim-2.2)$ of CPS stars, which would place the CPS progenitor among typical metallicities of ultra-faint dwarfs.

PAndAS MW stream: This stream, seen in panels 3 and 5 of Figure 3 , was found by Martin et al. (2014) in the PAndAS photometric survey of the area around M31. There is a wealth of Galactic substructure along the line of sight to M31 in this survey, including both TriAnd 1 and TriAnd 2, the Monoceros structure, and the Pisces/Triangulum stream. The width and velocity dispersion estimated by Martin et al. (2014) suggest a dwarf galaxy progenitor for the PAndAS stream. Stellar populations suggest that this is an old, metal-poor structure, and it follows an orbit that is roughly parallel to the Galactic plane at a distance of $\sim 17 \mathrm{kpc}$ from the Sun.

Styx: Styx is at a distance of $\approx 46 \mathrm{kpc}$ and is presumed to be a relic of a dwarf galaxy solely by virtue of its fairly substantial girth $\left(\sim 1^{\circ}\right)$ (Grillmair, 2009). A sparse but significant overdensity (Boötes III) is situated near the stream at a nearly identical distance, and Grillmair (2009) suggested that this overdensity might be the remains of the stream's progenitor. However, subsequent velocity measurements of Boötes III (Carlin et al., 2009) yielded $V_{\text {hel }}=197 \mathrm{~km} \mathrm{~s}^{-1}$, which appears to be at odds with the near perpendicularity of Styx to our line of sight. No velocities have yet been measured for the stream itself. 


\subsection{Clouds and other diffuse stellar structures}

Overdensities in Virgo: The nature and number of the substructure(s) in the Virgo constellation is uncertain. Main sequence turnoff (MSTO) stars from SDSS in this region of the sky have been used to show enhanced stellar densities spanning a huge sky area that have become known as the "Virgo Overdensity (VOD)." This was initially seen in a single stripe of SDSS imaging data at a distance of $\sim 20 \mathrm{kpc}$ by Newberg et al. (2002). Subsequent SDSS studies have mapped an overdensity (relative to neighboring regions of sky) spanning distances of at least $10<d<20 \mathrm{kpc}$ over $>1000 \mathrm{deg}^{2}$ (Jurić et al., 2008), and subsequently over $3000 \mathrm{deg}^{2}$ (Bonaca et al., 2012a). Overdensities of RR Lyrae stars are seen in the same region of sky, but in much smaller numbers. However, the much more precise distances that can be derived for RR Lyrae have led numerous authors to point out substructures localized in distance rather than in a large cloud-like structure as seen in MSTO stars. The first of these was the discovery by Vivas et al. (2001) of 5 RR Lyrae from the QUEST survey at a distance of $\sim 20 \mathrm{kpc}$; this was followed by a $\sim 19$ kpc clump in Vivas \& Zinn (2003), and a detection at $12<d<20 \mathrm{kpc}$ by Vivas \& Zinn (2006), also from QUEST. Subsequent RR Lyrae studies from the SEKBO survey by Keller et al. $(2008,2009)$ have seen similar overdensities at $\sim 16-20 \mathrm{kpc}$ distances. Additionally, Keller (2010) detected an overdensity between distances of $12<d<18 \mathrm{kpc}$ from the Sun using subgiants from SDSS.

While none of the photometric detections of Virgo substructures (see map in Figure 6) are completely inconsistent with each other, the patchiness among RR Lyrae detections suggests that there is at the very least some density variation within a single large structure, and perhaps a superposition of multiple structures that makes up the overall VOD. It is only with kinematics that this possibility can be explored. Follow-up spectroscopy of QUEST RR Lyrae initially showed (Duffau et al., 2006) that 6 stars apparently form a cold peak at $V_{\mathrm{gsr}} \approx 100 \mathrm{~km} \mathrm{~s}^{-1}$, which the authors dubbed the "Virgo Stellar Stream." Subsequent studies of this general region of sky (e.g., Newberg et al. 2007; Vivas et al. 2008; Prior et al. 2009; Brink et al. 2010; Carlin et al. 2012b) have variously found RV peaks between 100 and $250 \mathrm{~km} \mathrm{~s}^{-1}$ (see Figure 6), with some of these appearing to be localized in both distance and velocity. For example, Duffau et al. (2014) identified at least three peaks at distances ranging from $\sim 10$ to $20 \mathrm{kpc}$ and velocities between $\sim 120-220 \mathrm{~km} \mathrm{~s}^{-1}$. On the basis of such detections, it has been argued that the substructure in Virgo in made up of many tidal remnants overlapping in phase space. (Note, however, that Casey et al. 2012 found velocity peaks at $V_{\mathrm{GSR}} \sim 120$ and $\sim 200-240 \mathrm{~km} \mathrm{~s}^{-1}$. These authors identified the lower-velocity peak as the VSS, and speculate that the high-velocity feature may be related to the Sagittarius trailing tail.) Regardless of the origin, the radial velocities (see Figure 6) alone make it clear that the Virgo substructure is, on average, moving too rapidly away from the Galactic center to be a "cloud" of debris piling up at orbital apocenter as discussed in Chapter 6.

An important clue to the nature of the Virgo substructures came from the measurement of an orbit for Virgo stars in a single pencil-beam. Initially, Casetti-Dinescu et al. (2009) determined the orbit of a single RR Lyrae star known to be a Virgo mem- 


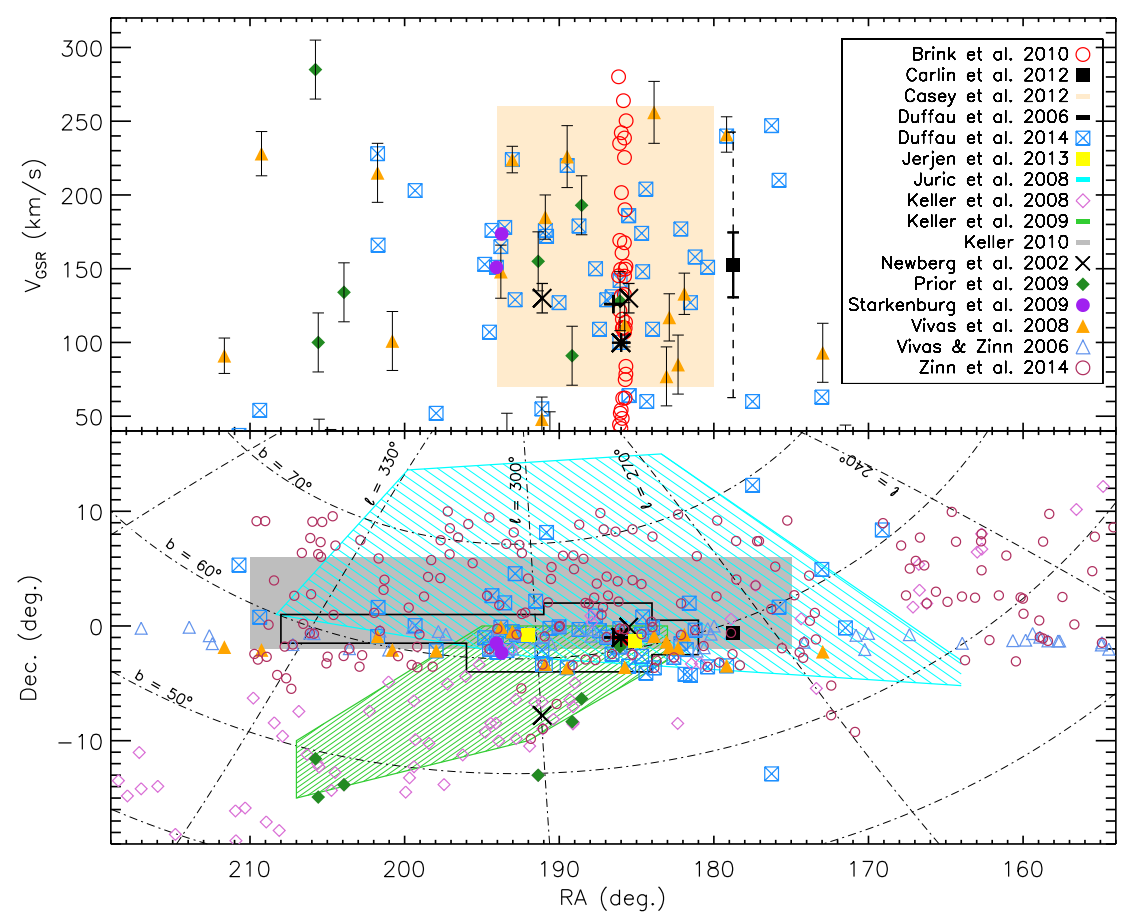

Fig. 6 Map of positions and velocities of stars that have been associated with the Virgo substructure(s) in the literature. The upper panel gives GSR-frame velocities as a function of right ascension, while the lower panel shows RA/Dec positions on the sky. Sources of the data are given in the legend; those with filled areas on the plot are given as horizontal lines of the same color as the fill. While there are many "excess" stars in this region of sky at velocities higher than expected for Milky Way populations, there is no clear spatial overdensity or kinematically cold peak visible in this figure.

ber. Their combined proper motions and radial velocity yield a rather eccentric orbit that suggests this star recently passed the pericenter of its orbit. This work was expanded by Carlin et al. (2012b), who measured proper motions and radial velocities of 17 stars consistent with VOD membership. This orbit confirms that the Virgo substructure results from an object on an eccentric orbit that recently made its closest approach. Indeed, Carlin et al. were able to qualitatively reproduce the large spatial extent (both on the sky and in distance) and velocity spread of the Virgo Overdensity by modeling a massive dwarf spheroidal disrupting on their measured orbit. This suggests that the entire structure may be a "puffed-up" dwarf galaxy that just passed its pericenter rather near the Galactic center. However, it is unclear how to reconcile the apparent clumpiness within the larger structure; this may be substructure related to group infall or structure within the progenitor satellite. Alternatively (as suggested by, e.g., Duffau et al. 2014), the entire structure may be a superposition of tidal structures overlapping each other on the sky, similar to the overlapping 
remnants that fell in along "preferred" directions in the simulations of Helmi et al. 2011. More observations and modeling are needed to understand the structures in this complicated region of the sky.

TriAnd1 and 2: Rocha-Pinto et al. (2004) identified an overdensity of 2MASS color-selected M-giant candidates between roughly $100^{\circ}<l<150^{\circ},-40^{\circ}<b<$ $-20^{\circ}$. This feature, dubbed the "Triangulum-Andromeda" (TriAnd) structure, is at heliocentric distances of $\sim 18-30 \mathrm{kpc}$. Rocha-Pinto et al. (2004) spectroscopically confirmed that TriAnd is a kinematically cold structure, and found a metallicity of $\langle[\mathrm{Fe} / \mathrm{H}]\rangle=-1.2$ with $\sim 0.5$-dex scatter. These authors also noted a trend of line-of-sight velocity with Galactic longitude that looks like an extension of the Monoceros velocity trend; however, TriAnd was thought to be too distant to be an extension of Monoceros. At nearly the same time, this same substructure was seen by Majewski et al. (2004) as a clear main sequence in the foreground of combined CMDs from their photometric survey of M31. These authors estimated the distance to be $\sim 16-25 \mathrm{kpc}$ from the Sun, with $\sim 20 \%$ higher distance at the furthest region from the Galactic plane compared to the lowest latitudes. The stellar density is roughly constant over the region surveyed, and is used to estimate a total luminosity over $\sim 1000 \mathrm{deg}^{2}$ of $\sim 5 \times 10^{5} L_{\odot}$.

A much deeper and larger-area survey of the M31 outskirts presented by Martin et al. (2007) revealed the existence of a second main sequence in the same region of the sky. This study, which covered $\sim 76 \mathrm{deg}^{2}$ between $\sim 115^{\circ}<l<130^{\circ},-30^{\circ}<b<$ $-25^{\circ}$, associated the brighter of the two MSTO features with TriAnd, and the fainter as a new structure dubbed "TriAnd2" (with the original structure called TriAnd1). These two features were found to be at heliocentric distances of $\sim 20$ and $\sim 28 \mathrm{kpc}$, and both show fairly narrow main sequences consistent with $\sim 2 \mathrm{kpc}$ line-of-sight depth. TriAnd1 shows stellar densities at the lowest latitudes (i.e., nearest the plane) and lowest longitudes in the region covered that are 2-3 $\times$ higher than at the opposite side of the survey footprint. The surface brightness $\left(\Sigma_{\mathrm{V}} \sim 32 \mathrm{mag} \mathrm{arcsec}{ }^{-2}\right)$ found by Martin et al. (2007) is similar to that derived by Majewski et al. (2004) for this very diffuse stellar substructure.

Sheffield et al. (2014) undertook an extensive spectroscopic survey of over $200 \mathrm{M}$ giant stars selected to be consistent with TriAnd membership. These authors found two distinct RGB features in 2MASS that correspond to the TriAnd1 and TriAnd2 MSTO features from Martin et al. (2007). Interestingly, even though the two features are separated by more than $5 \mathrm{kpc}$ in line-of-sight distance, their trends in lineof-sight velocity with Galactic longitude are indistinguishable. The nearer of these features (TriAnd1) is found by Sheffield et al. to have mean metallicity of $[\mathrm{Fe} / \mathrm{H}]$ $=-0.57$, while TriAnd 2 has $[\mathrm{Fe} / \mathrm{H}]=-0.64$. A possible origin for the two features is shown by these authors via an $N$-body model of a dwarf galaxy disrupting in the Milky Way halo. In this scenario, the two TriAnd features are debris that were stripped from a single satellite on consecutive pericentric passages of its orbit. The fairly large satellite mass required in this model is consistent with the metal-rich (relative to most dwarf galaxies) stellar populations in TriAnd; in order to enrich to such a level, the progenitor must have been fairly massive. 
The PAndAS deep photometric survey of the M31 vicinity has apparently resolved some wispy substructure on smaller scales in this region of sky. Martin et al. (2014) mapped stars from PAndAS in different distance slices, and found a narrow stream at a distance of $\sim 17 \mathrm{kpc}$, another wedge-shaped feature at $\sim 27 \mathrm{kpc}$ that is likely associated with TriAnd2, and wispy stellar structure throughout the $17-\mathrm{kpc}$ slice that is attributed to TriAnd 1 suffusing the entire field of view. Clearly there is a complex web of substructures intermingling in this region of the sky. Indeed, based on positional and kinematical similarities, Deason et al. (2014) argued in a recent analysis of spectroscopic data in this sky area that the PAndAS stream, TriAnd overdensities, and the Segue 2 ultrafaint dwarf galaxy are associated remnants of a group infall event. This scenario posits that the comparatively more metal-rich TriAnd represents the group-dominant central galaxy, with Segue 2 and the PAndAS structure remnants of TriAnd satellites. Finally, we note that Xu et al. (2015) recently suggested that TriAnd results from the oscillation of the midplane of the disk below $b=0^{\circ}$, and that the apparent stellar overdensity is part of a disk that extends out to $25 \mathrm{kpc}$ or more. There is clearly much more work needed to understand the nature of the TriAnd features.

Hercules-Aquila Cloud: This structure was originally discovered as an overdensity of MSTO stars in the SDSS DR5 database at heliocentric distances of $\sim 10-20 \mathrm{kpc}$ by Belokurov et al. (2007b). The cloud covers an enormous area of the sky, apparently extending to at least $\pm 40^{\circ}$ in Galactic latitude, centered on longitude of $l \sim 40^{\circ}$. Belokurov et al. attempted to identify the kinematical signature of the Cloud using SDSS spectra of RGB stars, and suggested that a peak at $V_{\mathrm{gsr}} \sim 180 \mathrm{~km} \mathrm{~s}^{-1}$ represents the velocity of Hercules-Aquila stars. Due to its similarity in the CMD to the SDSS ridgeline of the globular cluster M92 $([\mathrm{Fe} / \mathrm{H}]=-2.2)$, the stellar population of the Hercules-Aquila was suggested to be rather metal poor (perhaps slightly more metal-rich than M92).

In a series of papers, Larsen et al. $(2008,2011)$ extensively mapped a nearby structure between $\sim 1-6 \mathrm{kpc}$ from the Sun that was dubbed the "Hercules Thick Disk Cloud." The authors (Larsen et al. 2011) suggest that the overdensity seen by Belokurov et al. (2007b) was actually an artifact of the background-subtraction method used in that work, and that the true stellar overdensity is actually much more nearby. However, subsequent studies with unambiguous standard candles such as RR Lyrae have confirmed that there is a structure at $d_{\text {hel }} \sim 20 \mathrm{kpc}$. It remains unclear whether the more distant Hercules-Aquila cloud is related to the apparent thick disk extension.

Hercules-Aquila has been extensively mapped with RR Lyrae stars. Sesar et al. (2010a) used RR Lyrae from SDSS Stripe 82 (on the celestial equator in the south Galactic cap) to show that the Hercules-Aquila cloud contains at least 1.6 times the stellar density of the halo at $\sim 15-25 \mathrm{kpc}$. Photometric metallicity estimates from this work suggest that the cloud's mean metallicity is similar to that of the Galactic halo. Watkins et al. (2009) also studied the RR Lyrae in SDSS Stripe 82, using the proper motions of Bramich et al. (2008). Watkins and colleagues find 237 RR Lyrae that are likely associated with the Hercules-Aquila cloud; these actually make up the majority of their RR Lyrae star sample. The mean distance to the cloud in Stripe 82 
is estimated to be $\sim 22 \mathrm{kpc}$, but with dispersion (standard deviation) of $\sim 12 \mathrm{kpc}$. Watkins et al. estimate a mean photometric metallicity of $[\mathrm{Fe} / \mathrm{H}]=-1.43$ for the Hercules-Aquila stars. This study additionally presents the most reliable estimate of the velocity of this substructure. Stars with Hercules-Aquila cloud metallicities separate from the thick disk at $300^{\circ}<R A<320^{\circ}$ in velocity - the mean velocity is centered around $V_{\mathrm{gsr}} \approx 25 \mathrm{~km} \mathrm{~s}^{-1}$, with a long tail to lower velocities (and blending with the disk at higher velocities).

Simion et al. (2014) mapped the Hercules-Aquila cloud using RR Lyrae from the Catalina Sky Survey (Drake et al., 2014). After subtracting off the contribution of the underlying halo populations, this group concluded that the cloud is more prominent in the southern Galactic hemisphere than in the north, peaking at a heliocentric distance of $\sim 18 \mathrm{kpc}$. It is unclear whether the structure is truly asymmetric about the Galactic plane, or whether higher extinction in the north has affected the completeness of the RR Lyrae sample. The Hercules-Aquila RR Lyrae are predominantly of Oosterhoff class I; thus the stellar populations in the cloud are similar to those of the Galactic halo. The luminosity of the progenitor is estimated to be between $-15<M_{\mathrm{V}}<-9$, which would place it in the range of the brightest "classical" Milky Way dwarf galaxies.

Pisces Overdensity: The Pisces Overdensity is one of the most distant stellar overdensities known in the Galactic halo. This structure was originally found in a study of Stripe 82 RR Lyrae by Sesar et al. (2007) as a grouping of 26 stars (denoted by the authors as "clump J") at a mean distance of $81 \mathrm{kpc}$ from the Sun. This structure was confirmed by Watkins et al. (2009) in a separate analysis of RR Lyrae from SDSS Stripe 82. Watkins et al. dubbed the group of 28 stars at $\sim 80 \mathrm{kpc}$ mean distance the "Pisces Overdensity," and suggested that the total stellar mass of this substructure is $\sim 10^{4}-10^{5} \mathrm{M}_{\odot}$, with mean metallicity of $[\mathrm{Fe} / \mathrm{H}] \sim-1.5$. Kollmeier et al. (2009) obtained follow-up spectra of a handful of RR Lyrae stars in Stripe 82, and found five stars tightly clumped in velocity, confirming its identification as a coherent substructure that the authors suggested was a (possibly disrupted) dwarf galaxy. Additional spectra of RR Lyrae observed by Sesar et al. (2010b) complicated this picture by resolving the overdensity into two peaks separated in velocity by $\sim 100 \mathrm{~km} \mathrm{~s}^{-1}$. Thus the Pisces Overdensity may actually consist of more than one tidal debris feature overlapping in space. Sharma et al. (2010) subsequently found a much larger extended structure (dubbed "A16") in the same region of sky using 2MASS-selected M-giant candidates. In this work, the candidates are at distances of $\sim 100 \mathrm{kpc}$. The large spatial extent (including another possibly associated structure, "A14," at similar distances about 30 degrees away) and somewhat metal-rich (as evidenced by the presence of M-giants) nature of this structure was suggested to indicate an unbound satellite.

While the origin and nature of the Pisces Overdensity remains unclear, it is evident that there are tidal debris structures at distances of more than $80 \mathrm{kpc}$ in the halo. Further kinematical and chemical analysis will be essential to relate the Pisces substructure to other known satellites or structures in the halo.

Perseus Cloud: Another poorly studied apparent substructure is the Perseus Cloud, originally isolated among 2MASS-selected M-giant candidates by Rocha- 
Pinto et al. (2004). Though it appears to be an extension of the TriAnd structure on the sky, and is at a similar distance, Rocha-Pinto et al. argue that their spectroscopic velocities show the two structures to be unrelated. The nature of this stellar structure remains undetermined.

Canis Major and Argo: There are other purported stellar overdensities in the Milky Way that we have not discussed here because their nature (as an actual overdensity and/or as a tidal debris structure) is still undetermined. These include the Canis Major overdensity, which was originally seen as a low-latitude excess of 2MASS-selected M giants by Martin et al. (2004a). This feature has been suggested to be a recently accreted dwarf galaxy (Martin et al., 2004a b; Bellazzini et al., 2006a), and perhaps even the progenitor of the Monoceros Ring (e.g., Peñarrubia et al. 2005). Others (including Momany et al. 2006, Moitinho et al. 2006) have attributed this apparent stellar overdensity to the warp and flare of the Galactic disk. The Argo stellar overdensity (Rocha-Pinto et al. 2006) is near the Canis Major feature in 2MASS M giants, and is also poorly studied. As the focus of this review is on known tidal substructures, and the nature of these structures is still under debate, we will not discuss them further. Some discussion of Canis Major and its possible relation to the low-latitude Monoceros structure can be found in Chapter 3 of this volume.

\section{Future Discoveries}

Given that the currently available, large-area digital sky surveys do not cover the entire sky, we expect that a significant number of strong streams remain to be discovered within $50 \mathrm{kpc}$. The Pan-STARRS survey (Kaiser et al. 2002), which covers considerably more sky area than the SDSS, will presumably yield additional streams, and extend at least some of the streams whose known extent is currently limited by the SDSS footprint. In the southern hemisphere, the ATLAS (Shanks et al. 2013), Skymapper (Keller et al. 2007), and Dark Energy Surveys (Rossetto et al. 2011) remain to be completed. Covering regions of the sky that have been largely unexplored to date, they will almost certainly yield a number of new streams, in addition to extending some of the streams and clouds in Tables 1 and 2. We note that extending known streams is at least as important as finding new ones, as longer streams both reduce the uncertainty in orbit shape and tighten the possible constraints on the Galactic potential.

Further progress in finding cold or tenuous streams in the SDSS and other surveys will almost certainly result from simultaneously combining photometric filtering with velocity and proper motion measurements. Spectra are now available for $\sim 7 \times 10^{5}$ stars in the SDSS DR10. Newberg et al. (2009) combined photometric measurements with velocities and metallicities to detect the Cetus Polar Stream, a structure which had eluded prior discovery through photometric means alone. Several spectroscopic surveys either planned or in progress (e.g., LAMOST) will almost 
certainly make contributions in this area, particularly in detecting streams that are highly inclined to our line of sight (e.g., ECHOS, Schlaufman et al. 2009).

The Large Synoptic Survey, expected to begin early in the next decade, will greatly extend the reach of current photometric (e.g., matched filter) methods of stream detection. Even a single pass over the visible sky will be significantly deeper than the SDSS, and the end-of-survey photometric depth is expected to reach $r=27.5$ (Grillmair \& Sarajedini 2009). On the other hand, background galaxies will vastly outnumber stars at this depth, and significant improvements will need to made in the area of star-galaxy separation if we are to take full advantage of the data. If this can be achieved, then applying matched-filter techniques to upper main sequence stars should enable us to photometrically detect streams out to as much as $500 \mathrm{kpc}$. RR Lyrae will enable us to probe even more deeply into the halo (e.g. Sesar et al. 2013); even with fairly conservative single-pass detection limits, LSST should enable us to detect RR Lyrae out to more than $700 \mathrm{kpc}$.

Distance estimates are currently the largest source of uncertainty in the use of tidal streams as probes of the potential. The Gaia survey will clearly have an enormous impact here, providing trigonometric distances for giant branch stars out to tens of kpc. In addition, a number of synoptic surveys have detected many thousands of RR Lyrae, some of which have been associated with known streams (e.g. Sesar et al. 2013), and whose distances can now be measured to $\simeq 2 \%$ using infrared photometry. On the other hand, the coldest streams may have very few giant branch stars or RR Lyrae. Distances for these have often been estimated photometrically, using filter matching or upper main sequence fitting. However, the ages and metallicities of the stream stars are only poorly known, and the distance uncertainties due to inappropriate filter templates are correspondingly large. Eyre \& Binney (2009) and Eyre (2010) have described and demonstrated an alternative method of finding distances by using proper motions to measure "Galactic parallax." This technique relies on the premise that any net proper motion component of stars perpendicular to a stream must be a consequence of the sun's reflex motion. The accuracy of the method is currently on par with photometric distances, limited primarily by the quality of the available proper motions. The Gaia survey should enable very significant improvements in the application of this technique.

Proper motions can also be used to verify and even detect streams, and both Gaia and LSST are expected to make significant contributions to the field. With expected end-of-mission proper motion accuracies of $\leq 200 \mu$ as to 20th magnitude, the Gaia survey will enable us to push well down the stream mass function out to perhaps tens of kpc. Beyond that, the end-of-survey proper motion uncertainties for LSST will be on the order of $100 \mathrm{~km} \mathrm{~s}^{-1}$ at $100 \mathrm{kpc}$. With suitable averaging and the application of Bayesian techniques, this should be sufficient to detect the remote and particularly high-value, extended tidal tails of many of the dwarf galaxies.

Acknowledgements JLC gratefully acknowledges support from the NSF under grants AST 0937523 and AST 14-09421. 


\section{References}

Alard, C. 1996, ApJ Lett, 458, L17

Bellazzini, M., Ibata, R., Martin, N., Lewis, G. F., Conn, B., \& Irwin, M. J. 2006a, MNRAS, 366, 865

Bellazzini, M., Newberg, H. J., Correnti, M., Ferraro, F. R., \& Monaco, L. 2006b, A\&A, 457, L21

Belokurov, V., Evans, M. W., Irwin, M. J., et al. 2006a, ApJ, 637, L29

Belokurov, V., Zucker, D. B., Evans, N. W., et al. 2006b, ApJ Lett, 642, L137

Belokurov, V., Evans, N. W., Irwin, M. J., et al. 2007a, ApJ, 658, 337

Belokurov, V., Evans, N. W., Bell, E. F., et al. 2007b, ApJ Lett, 657, L89

Bernard, E. J., Ferguson, A. M. N., Richardson, J. C., et al. 2014, MNRAS, 443, 84

Bonaca, A., Jurić, M., Ivezić, Ž., et al. 2012a, AJ, 143, 105

Bonaca, A., Geha, M., \& Kallivayalil, N. 2012b, ApJ Lett, 760, LL6

Bramich, D. M., Vidrih, S., Wyrzykowski, L., et al. 2008, MNRAS, 386, 887

Brink, T. G., Mateo, M., \& Martínez-Delgado, D. 2010, AJ, 140, 1337

Carlberg, R. G. 2009, ApJ Lett, 705, L223

Carlberg, R. G.,Grillmair, C. J., \& Hetherington, N. 2012, ApJ, 760, 75

Carlberg, R. G. \& Grillmair, C. J. 2013, ApJ, 768, 171

Carlin, J. L., Grillmair, C. J., Muñoz, R. R., Nidever, D. L., \& Majewski, S. R., 2009, ApJ, 702, 9

Carlin, J. L., Majewski, S. R., Casetti-Dinescu, D. I., Law, D. R., Girard, T. M., \& Patterson, R. J. 2012a, ApJ, 744, 25

Carlin, J. L., Yam, W., Casetti-Dinescu, D. I., et al. 2012b, ApJ, 753, 145

Casetti-Dinescu, D. I., Girard, T. M., Majewski, S. R., et al. 2009, ApJ Lett, 701, L29

Casey, A. R., Keller, S. C., \& Da Costa, G. 2012, AJ, 143, 88

Casey, A. R., Da Costa, G., Keller, S. C., \& Maunder, E. 2013, ApJ, 764, 39

Casey, A. R., Keller, S. C., Da Costa, G., Frebel, A., \& Maunder, E. 2014, ApJ, 784, 19

Chou, M.-Y., Majewski, S. R., Cunha, K., et al. 2007, ApJ, 670, 346

Chou, M.-Y., Majewski, S. R., Cunha, K., Smith, V. V., Patterson, R. J., \& MartinezDelgado, D. 2011, ApJ, 731, L30

Cui, X.-Q., Zhao, Y.-H., Chu, Y.-Q., et al. 2012, Research in Astronomy and Astrophysics, 12, 1197

Deason, A. J., Belokurov, V., Hamren, K. M., et al. 2014, MNRAS, 444, 3975

Dehnen, W., Odenkirchen, M., Grebel, E. K., \& Rix, H.-W. 2004, AJ, 127, 2753

Deng, L.-C., Newberg, H. J., Liu, C., et al. 2012, Research in Astronomy and Astrophysics, 12, 735

Drake, A. J., Graham, M. J., Djorgovski, S. G., et al. 2014, ApJS, 213, 9

Duffau, S., Zinn, R., Vivas, A. K., et al. 2006, ApJ Lett, 636, L97

Duffau, S., Vivas, A. K., Zinn, R., Méndez, R. A., \& Ruiz, M. T. 2014, A\&A, 566, AA118

Eyre, A. 2010, MNRAS, 403, 1999

Eyre, A., \& Binney, J. 2009, MNRAS, 399, L160 
Fellhauer, M., Belokurov, V., Evans, N. W., et al. 2006, ApJ, 651, 167

Fellhauer, M., Evans, N. W., Belokurov, V., et al. 2007b, MNRAS, 375, 1171

Fellhauer, M., Evans, N. W., Belokurov, V., Wilkinson, M. I., \& Gilmore, G. 2007a, MNRAS, 380, 749

Grillmair, C. J., 2006a, ApJ, 645, L37

Grillmair, C. J., 2006b, ApJ, 651, L29

Grillmair, C. J. 2009, ApJ, 693, 1118

Grillmair, C. J. 2011, ApJ, 738, 98

Grillmair, C. J. 2014, ApJ, 790, 10

Grillmair, C. J., Freeman, K. C., Irwin, M., \& Quinn, P. J. 1995, AJ, 109, 2553

Grillmair, C. J., \& Johnson, R. 2006, ApJ, 639, L17

Grillmair, C. J., \& Dionatos, O. 2006a, ApJ, 641, L37

Grillmair, C. J., \& Dionatos, O. 2006b, ApJ, 643, L17

Grillmair, C. J., Carlin, J. L., \& Majewski, S. R. 2008, ApJ, 689, L117

Grillmair, C. J., \& Sarajedini, A. LSST Science Book, LSST Corporation, Tucson (2009)

Grillmair, C. J., Cutri, R., Masci, F. J., et al. 2013, ApJ, 769, 23

Helmi, A., Cooper, A. P., White, S. D. M., et al. 2011, ApJ Lett, 733, L7

Ibata, R. A., Gilmore, G., \& Irwin, M. J. 1994, Nature, 370, 194

Ibata, R., Lewis, G. F., Irwin, M., Totten, E., \& Quinn, T. 2001a, ApJ, 551, 294

Ibata, R., Irwin, M., Lewis, G. F., \& Stolte, A. 2001b, ApJ Lett, 547, L133

Ibata, R. A., Irwin, M. J., Lewis, G. F., Ferguson, A. M. N., \& Tanvir, N. 2003, MNRAS, 340, L21

Irwin, M., \& Hatzidimitriou, D. 1995, MNRAS, 277, 1354

Jerjen, H., Da Costa, G. S., Willman, B., et al. 2013, ApJ, 769, 14

Jurić, M., Ivezić, Ž., Brooks, A., et al. 2008, ApJ, 673, 864

Kaiser, N., Aussel, H., Burke, B., et al. 2002, SPIE, 4836, 154

Keller, S. C., Schmidt, B. P., Bessell, M. S., et al. 2007, PASA, 24, 1

Keller, S. C., Murphy, S., Prior, S., Da Costa, G., \& Schmidt, B. 2008, ApJ, 678, 851

Keller, S. C., da Costa, G. S., \& Prior, S. L. 2009, MNRAS, 394, 1045

Keller, S. C., Yong, D., \& Da Costa, G. S. 2010, ApJ, 720, 940

Kollmeier, J. A., Gould, A., Shectman, S., et al. 2009, ApJ Lett, 705, L158

Koposov, S., Rix, H-W, \& Hogg, D. W. 2010, ApJ, 712, 260

Koposov, S., Irwin, M., Belokurov, V., Ganzalez-Solares, E., Kupcu, Y.A., Lewis, J., Metcalfe, N., \& Shanks, T. 2012, ApJ, 750, 80

Koposov, S. E., Irwin, M., Belokurov, V., et al. 2014, MNRAS, 442, L85

Küpper, A. H. W., MacLeod, A., \& Heggie, D. C. 2008, MNRAS, 387, 1248

Küpper, A. H. W., Lane, R. R., \& Heggie, D. C. 2012, MNRAS, 420, 2700

Kuzma, P. B., Da Costa, G. S., Keller, S. C., \& Maunder, E. 2015, MNRAS, 446, 3297

Larsen, J. A., Humphreys, R. M., \& Cabanela, J. E. 2008, ApJ Lett, 687, L17

Larsen, J. A., Cabanela, J. E., \& Humphreys, R. M. 2011, AJ, 141, 130

Law, D. R., Majewski, S. R., \& Johnston, K. V. 2009, ApJ Lett, 703, L67

Law, D. R., \& Majewski, S. R. 2010, ApJ, 714, 229 
Leon, S., Meylan, G., \& Combes, F. 2000, A\&A, 359, 907

Li, J., Newberg, H. J, Carlin, J. L, et al. 2012, ApJ, 757, 151

Luo, A.-L., Zhang, H.-T., Zhao, Y.-H., et al. 2012, Research in Astronomy and Astrophysics, 12, 1243

Lux, H., Read, J. I., Lake, G., \& Johnston, K. V. 2012, MNRAS, 424, 16

Majewski, S. R., Skrutskie, M. F., Weinberg, M. D., \& Ostheimer, J. C. 2003, ApJ, 599, 1082

Majewski, S. R., Ostheimer, J. C., Rocha-Pinto, H. J., et al. 2004, ApJ, 615, 738

Martin, C., Carlin, J. L., Newberg, H. J., \& Grillmair, C. J., 2013, ApJ, 765, 39

Martin, N. F., Ibata, R. A., Bellazzini, M., Irwin, M. J., Lewis, G. F., \& Dehnen, W. 2004a, MNRAS, 348, 12

Martin, N. F., Ibata, R. A., Conn, B. C., Lewis, G. F., Bellazzini, M., Irwin, M. J., \& McConnachie, A. W. 2004b, MNRAS, 355, L33

Martin, N. F., Ibata, R. A., \& Irwin, M. 2007, ApJ, 668, L123

Martin, N. F., Ibata, R. A., Rich, R. M., et al. 2014, ApJ, 787, 19

Martínez-Delgado, D., Gómez-Flechoso, M. Á., Aparicio, A., \& Carrera, R. 2004, ApJ, 601, 242

Mastrobuono-Battisti, A., Di Matteo, P., Montuori, M., \& Haywood, M. 2012, A\&A, 546, LL7

Mateo, M., Olszewski, E. W., \& Morrison, H. L. 1998, ApJ Lett, 508, L55

Moitinho, A., Vázquez, R. A., Carraro, G., et al. 2006, MNRAS, 368, L77

Momany, Y., Zaggia, S., Gilmore, G., et al. 2006, A\&A, 451, 515

Newberg, H. J., Yanny, B., Rockosi, C., et al. 2002, ApJ, 569, 245

Newberg, H. J., Yanny, B., Cole, N., et al. 2007, ApJ, 668, 221

Newberg, H. J., Yanny, B., \& Willett, B. A. 2009, ApJ, 700, L61

Newberg, H. J., Willett, B. A., Yanny, B., \& Xu, Y. 2010, ApJ, 711, 32

Odenkirchen, M., Grebel, E. K., Rockosi, C. M., et al. 2001, ApJ, 548, L165

Odenkirchen, M., Grebel, E. K., Dehnen, W., et al. 2003, AJ, 126, 2385

Odenkirchen, M., Grebel, E. K., Kayser, A., Rix, H.-W., \& Dehnen, W. 2009, AJ, 137, 3378

Peñarrubia, J., Martínez-Delgado, D., Rix, H. W., et al. 2005, ApJ, 626, 128

Prior, S. L., Da Costa, G. S., Keller, S. C., \& Murphy, S. J. 2009, ApJ, 691, 306

Rocha-Pinto, H. J., Majewski, S. R., Skrutskie, M. F., \& Crane, J. D. 2003, ApJ, 594, L115

Rocha-Pinto, H. J., Majewski, S. R., Skrutskie, M. F., Crane, J. D., \& Patterson, R. J. 2004, ApJ, 615, 732

Rocha-Pinto, H. J., Majewski, S. R., Skrutskie, M. F., Patterson, R. J., Nakanish, H., Munoz, R. R., \& Sofue, Y. 2006, ApJ, 640, L150

Rockosi, C. M., Odenkirchen, M., Grebel, E. K., et al. 2002, AJ, 124, 349

Rossetto, B. M., Santiago, B. X., Girardi, L., et al. 2011, AJ, 141, 185

Sales, L. V., Helmi, A., Starkenburg, E., et al. 2008, MNRAS, 389, 1391

Schlaufman, K. C., Rockosi, C. M., Allende Prieto, C., et al. 2009, ApJ, 703, 2177

Sesar, B., Ivezić, Ž., Lupton, R. H., et al. 2007, AJ, 134, 2236

Sesar, B., Ivezić, Ž., Grammer, S. H., et al. 2010a, ApJ, 708, 717

Sesar, B., Vivas, A. K., Duffau, S., \& Ivezić, Ž. 2010b, ApJ, 717, 133 
Sesar, B., Grillmair, C. J., Cohen, J. G., et al. 2013, ApJ 776, 26

Sesar, B., Bovy, J., Bernard, E. J., et al. 2015, ApJ, submitted; arXiv:1501.00581

Shanks, D. J., Belokurov, V., Chehade, B., et al. 2013, The Messenger, 154, 38

Sharma, S., Johnston, K. V., Majewski, S. R., et al. 2010, ApJ, 722, 750

Sheffield, A. A., Johnston, K. V., Majewski, S. R., et al. 2014, ApJ, 793, 62

Simion, I. T., Belokurov, V., Irwin, M., \& Koposov, S. E. 2014, MNRAS, 440, 161

Slater, C. T., Bell, E. F., Schlafly, E. F., et al. 2014, ApJ, 791, 9

Starkenburg, E., Helmi, A., Morrison, H. L., et al. 2009, ApJ, 698, 567

Totten, E. J., \& Irwin, M. J. 1998, MNRAS, 294, 1

Vivas, A. K., Zinn, R., Andrews, P., et al. 2001, ApJ Lett, 554, L33

Vivas, A. K., \& Zinn, R. 2003, Memorie della Societa Astronomica Italiana, 74, 928

Vivas, A. K., Zinn, R., Gallart, C. 2005, AJ, 129, 189

Vivas, A. K., \& Zinn, R. 2006, AJ, 132, 714

Vivas, A. K., Jaffé, Y. L., Zinn, R., et al. 2008, AJ, 136, 1645

Vivas, A. K., Sesar, B., Duffau, S., \& Ivezic, Z. 2011, Revista Mexicana de Astronomia y Astrofisica Conference Series, 40, 261

Watkins, L. L, Evans, N. W., Belokurov, V., et al. 2009, MNRAS, 398, 1757

Willett, B. A., Newberg, H. J., Zhang, H., Yanny, B., \& Beers, T. C. 2009, ApJ, 697, 207

Xu, Y., Newberg, H. J., Carlin, J. L., et al. 2015, ApJ, accepted

Yam, W., Carlin, J. L., Newberg, H. J., Dumas, J., O’Malley, E., Newby, M., \& Martin, C. 2013, ApJ, 776, 133

Yanny, B., Newberg, H. J., Grebel, E. K., et al. 2003, ApJ, 588, 824

Yanny, B., Newberg, H. J., Johnson, J. A., et al. 2009, ApJ, 700, 1282

Zhao, G., Zhao, Y.-H., Chu, Y.-Q., Jing, Y.-P., \& Deng, L.-C. 2012, Research in Astronomy and Astrophysics, 12, 723

Zinn, R., Horowitz, B., Vivas, A. K., et al. 2014, ApJ, 781, 22 